\title{
Search for Evidence of Radiative Equilibrium Departures in Extended Atmospheres
}

\author{
Roberto Venero ${ }^{1}$; Lydia Cidale ${ }^{2}$ and Adela Ringuelet ${ }^{2}$ \\ Facultad de Ciencias Astronómicas y Geofísicas, Universidad Nacional \\ de La Plata, Paseo del Bosque S/N, 1900 - La Plata, Argentina
}

\begin{abstract}
Through the analysis of line profiles of atoms in different ionization stages, we study the source function behavior of those elements that allow us to outline temperature structures in moving extended atmospheres. The radiative transfer equation is solved in the comoving frame and NLTE for multilevel atoms.
\end{abstract}

\section{Introduction}

Early type stars have extended moving atmospheres where complex phenomena give rise to the observed spectral features. Departures of radiative and hydrostatic equilibrium in the atmospheres of these stars are very important as they control the shape and strength of the line profiles. Many of these characteristics have been successfully reproduced applying models with temperature structures that resemble a chromosphere. Some of these results are the $\mathrm{H} \alpha$ profile in $\mathrm{AB}$ Aur (Catala \& Kunasz, 1987), the shape of the $\mathrm{H}$ emission in Be stars (Cidale \& Ringuelet 1993), the Mg II lines (Cidale, 1998) and the IR hydrogen lines (Cidale et al. 1999). Following the basic ideas of the previously mentioned papers, we computed the line profiles of $\mathrm{He}$ II, Si IV and Ca II for different atmospheric models in order to understand which mechanism prevails in the line formation process and where it is taking place. Here, we will present some results for $\mathrm{Si}$ IV line profiles. Under the conditions of the studied models, the computed lines of Ca II $\lambda \lambda 3933,3968 \AA$ were extremely faint. The work devoted to the study of He II $\lambda 1640 \AA$ is going to be analyzed later.

\section{The Model}

We performed the calculation of line profiles in spherically extended atmospheres in expansion. This simple configuration allows us to discriminate between those features in the profiles due to the velocity and temperature structures and those due to the model geometry. Moreover, He II and Si IV formation regions may be quite spherical since there is no evidence of correlation between $V \sin (i)$ and

\footnotetext{
${ }^{1}$ Fellow of Fomec, Ministerio de Educación, Argentina

${ }^{2}$ Member of the Carrera del Investigador Científico, CONICET, Argentina
} 

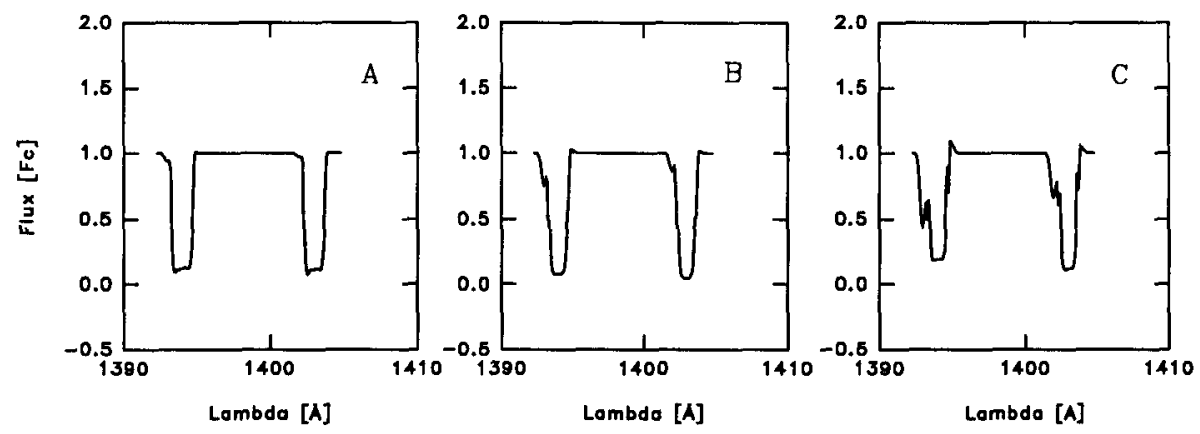

Figure 1. Resonance Si IV lines for different chromospheric locations

the equivalent width of Si IV (Snow \& Stalio 1987) and He II (Peters 1998) lines. Here we analyzed models with $T_{\text {eff }}=25000 \mathrm{~K}$ and $\log g=3.5$. The atmosphere is composed of spherical shells with different thermodynamic states. The deepest one is the photosphere, in hydrostatic equilibrium, represented by the corresponding Kurucz's model (1979). Above the photosphere the temperature rises as a consequence of non-radiative fluxes originating, therefore, a chromosphere where the velocity increases reaching there, in some cases, the terminal velocity. Finally, the temperature decreases towards an isothermal value in a very low density shell. In this case, the temperature structure is similar to that used by Catala \& Kunasz (1987); model atmospheres without chromospheres are analyzed as well. The wind is represented with a monotonically increasing velocity law characterized by the terminal velocity and the slope of the innermost positive gradient region. The results showed here correspond to softly increasing velocity laws with mass loss rates that range from $1.510^{-9}$ to $1.010^{-7} \mathrm{M}_{\odot} \mathrm{yr}^{-1}$.

We used a simplified atomic model of Si IV in order to study the resonance lines. The atom was modelled with 6 levels from 3 s to $4 \mathrm{~d}$ and one level for $\mathrm{Si} \mathrm{V}$. Ionization thresholds and oscillator strength were taken from Becker \& Butler (1990). The transfer and the statistical equilibrium equations were solved selfconsistently in the comoving frame (Mihalas \& Kunasz 1978). Starting with LTE distributions, the populations and the radiation field are updated iteratively by means of the equivalent two-level atom approach (ETLA). We have determined the line forming regions integrating the radiative flux along impact parameters and studying the amount of its contribution to the overall radiative flux at the line wavelength. The knowledge of the region contributing to the line formation may give us clues to understand some peculiarities in the line profile.

\section{Main Results}

We studied many models resulting from the combination of temperature structures and velocity laws. A high temperature regime along the extended atmosphere is needed in order to obtain the Si IV resonance lines. Models with low temperature envelopes tend to depopulate the ion and to slow down the convergence rate of the equations. 


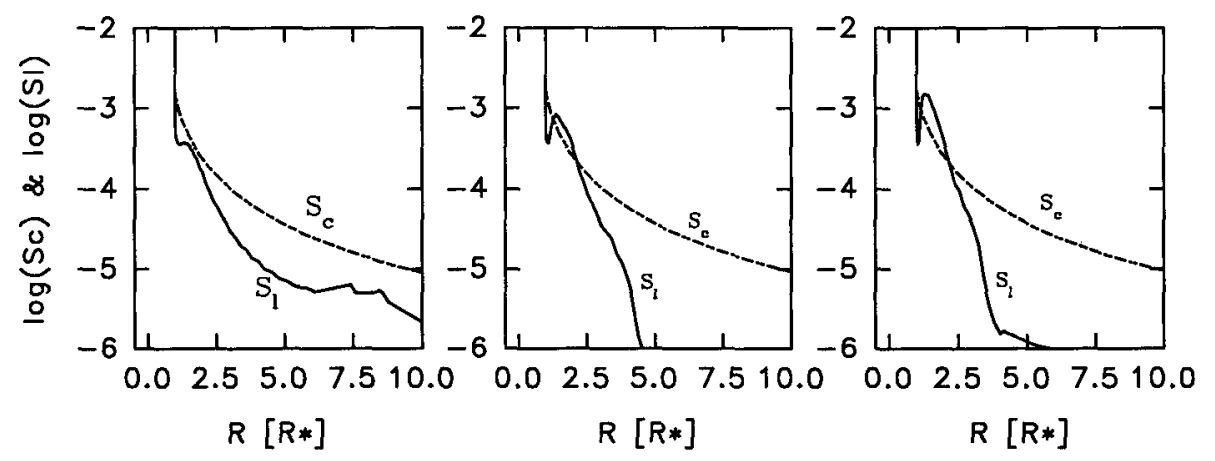

Figure 2. Continuum and line source functions corresponding to Figure $1(\mathrm{~A}),(\mathrm{B})$, and $(\mathrm{C})$, respectively

Fig. 1 (A) displays the doublet Si IV $\lambda \lambda 1394,1403 \AA$ for a chromospheric model with a maximum temperature, $T_{\max }=50,000 \mathrm{~K}$, located at $6 R_{*}$, an isothermal envelope with $T_{\mathrm{o}}=10,000 \mathrm{~K}$ and a terminal velocity of $250 \mathrm{~km}$ $\mathrm{s}^{-1}$. The lines are strong; some emission seems to fill their cores and the wings look quite symmetric. Significant changes in these profiles are obtained when the location of the chromospheric temperature maximum is shifted towards the star. Profiles of Figs. 1 (B) and 1 (C) correspond to models with $T_{\max }$ placed at 3 and $2 R_{*}$, respectively. A blue component appears and increases in intensity as the chromosphere is placed closer to the photosphere. In Fig. 1 (C) more than one component is present.

From the analysis of the emergent radiation along several impact parameters we note that the position of the forming region is slightly modified when the chromosphere is shifted and roughly corresponds to the intersection of the temperature rise and the density drop. The strongest blue-shifted narrow component receives the maximum contribution from the central impact parameter and its velocity approximately matches the terminal velocity of the wind. In a region between the photosphere and the chromosphere the population of the $\mathrm{Si}$ IV ion reaches the maximum value. As the density of the region decreases outward, the population of Si IV decreases too and the amount of Si V atoms rises as far as the temperature of the chromosphere increases. At a certain point the situation is reversed: Si V recombines into the ground level of Si IV. We propose that this region originates the strongest blueshifted narrow component. The low velocity gradients, in the region close to the terminal velocity, favour the formation of a narrow component. Fig. 2 illustrates the run of the continuum source function $S_{c}$ (calculated at the line wavelength) and the line source function $S_{l}$ for the models mentioned above. $S_{l}$ is collisionally dominated at the beginning of the chromosphere since it follows the temperature rise and then, is radiative dominated due to the decrease in density. Higher values of $S_{l}$ are obtained when the chromospheres is closer to the star.

Fig. 3 exhibits Si IV $\lambda \lambda 1394,1403 \AA$ for a simple model (without chromosphere) consisting in a photosphere and an isothermal envelope. Fig. 3 (A) was computed with a smooth velocity gradient near the photosphere and Fig. 3 (B) 

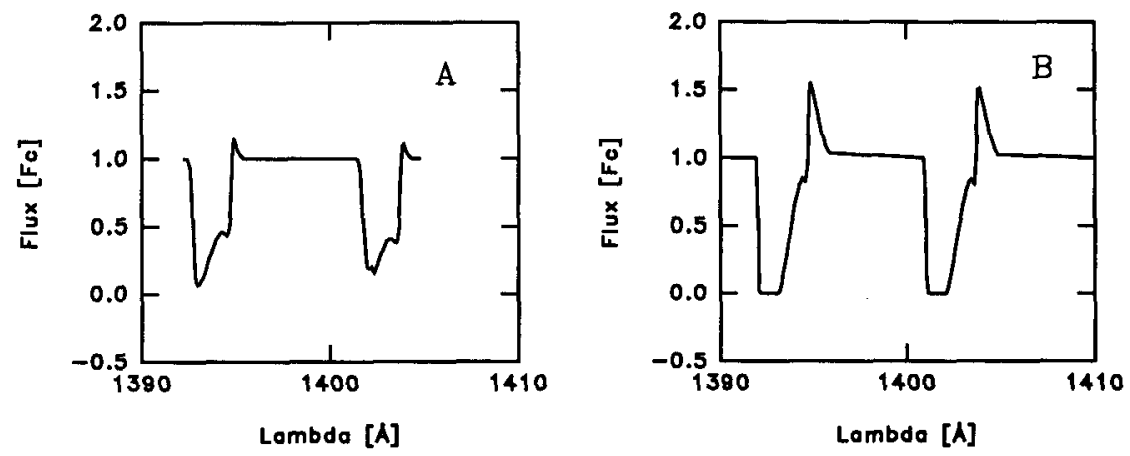

Figure 3. Profiles computed for models without a chromosphere

with a steeper velocity gradient and higher terminal velocity. The lines look asymmetric and $\mathrm{P}$ Cygni features are evident.

\section{Conclusions}

Si IV $\lambda \lambda 1394,1403 \AA$ profiles were obtained for atmospheric models with radiative equilibrium departures. High temperature structures are needed in order to obtain appropriate Si IV populations. As the Si IV lines are observed in stars even cooler than those we discuss (Marlborough \& Peters 1986), more plausible mechanisms (as charge transfer reactions) should be included to populate these ions. The Si IV lines we calculated are sensitive to high temperature structures such as a chromosphere. The appearance of displaced narrow components in the line profile may be due to the presence of a chromosphere in the deepest regions of the atmosphere. The lines receive an important contribution from a region located around $2 R_{*}$. Line profiles from models without a chromosphere are strongly controlled by the velocity law and display $P$ Cygni features.

\section{References}

Becker, S.R., Butler, K. 1990, å 235, 326.

Catala, C., Kunasz, P.B. 1987, å 134, 402.

Cidale, L.S., Ringuelet, A.E. 1993, ApJ 411, 874.

Cidale, L.S. 1998, ApJ 502, 824.

Cidale, L.S. 1999, IAU Coll. 175

Marlborough, J.M., Peters, G. 1987 in IAU Symp. 98, 387.

Kurucz, R.L. 1979, ApJS 40, 1.

Mihalas, D., Kunasz, P.B. 1978, ApJ 219, 635.

Peters, G.J. 1998, in Angular Momentum and Mass Loss for Hot Stars, Wilson L.A., Stalio R. eds, Kluwer Academic Publishers, p. 219

Snow, T.P., Stalio R. 1987, in Exploring the Universe with the IUE satellite, Kondo Y. ed., Reidel Publishing Company, p. 183 